\title{
Horse Crazy - Gefühle im Konsumkapitalismus
}

blog.kulturwissenschaften.de/pferdemaedchen/

\section{Anja SchwanhäußerMensch und Tier}

Das Pferdemädchen, jene oft belächelte Figur, die für eine mädchenhafte Leidenschaft in der Adoleszenz steht, erhält in jüngerer Zeit einige Aufmerksamkeit. Und dies nicht nur auf dem Jugendmarkt, der seit über einem halben Jahrhundert einschlägige Romane, Filme und Zeitschriften hervorbringt, sondern auch in der Hochkultur. Der Historiker Ulrich Raulff widmet ihnen ein Kapitel seiner umfassenden Kulturgeschichte „Das letzte Jahrhundert der Pferde. Geschichte einer Trennung“1, und die Schriftstellerin Juli Zeh verfasst eine „Gebrauchsanweisung für Pferde“, in der sie eingangs bekennt: „Ich war ein echtes Pferdemädchen.“2 Auch der Jenaer Kunstverein eröffnete im Sommer 2020 die Ausstellung „Dressuren der Durchlässigkeit“ (kuratiert von Lucia Groß) und scheut im Katalog nicht vor ästhetischen Referenzen zur Zeitschrift „Wendy“ und dem ausgiebigen Einsatz der Farbe Pink zurück. Weitere Beispiele ließen sich anführen. Damit wird „a little girl's horse craze“ 3 , wie Anna Freud ihn nannte, salonfähig. Wie ist dieser neuerliche Trend $\mathrm{zu}$ verstehen?

\section{Gefühlsstruktur im Konsumkapitalismus}

Die nahe liegende Erklärung ist, dass einstige Pferdemädchen nun erwachsen und einflussreich geworden sind und die Themensetzung im Kulturbetrieb mitbestimmen. Doch steht der Trend mit breiteren gesellschaftlichen Entwicklungen in Beziehung. Denn Pferde und Mädchen erweisen sich im Konsumkapitalismus ${ }^{4}$ als Spiegel und Agens zeitgenössischer Gefühlsstruktur: An ihnen hat nicht nur der Wandel der Geschlechterbeziehungen seine Spuren hinterlassen. Ganz allgemein katalysieren Pferde und Mädchen drängende Gesellschaftsfragen wie Umweltbewusstsein und soziale Verantwortung. Denn Werte wie Care und Rücksichtnahme sowie die Frage nach dem sozialen Miteinander sind nicht nur typische Pferdemädchen-Probleme, sie stehen ganz allgemein im Zentrum aktueller gesellschaftlicher Konfliktlinien und sozialer Proteste. Hinzu kommt, dass gesellschaftliche Themen zunehmend mit und durch die Pop- und Populärkultur, das heißt, ästhetisch verhandelt werden. Dabei ist die Unmöglichkeit sinnlicher und lustvoller Beziehungen nicht erst seit Ausbruch der Pandemie ein notorisches Problem.

\section{Coming-out}

Innerhalb dieser Gefühlsstruktur wird das konventionell weibliche Stereotyp des Pferdemädchens hinterfragt. Raulff kritisiert den populären Mythos, wonach Pferde für Mädchen eine Art Ersatzobjekt beim Übergang von der Jugend ins Erwachsenenalter darstellten. Über ihm hänge „der Richtungspfeil der Teleologie“5. 
Als Gegenbeispiel verweist er auf den queeren Film „Von Mädchen und Pferden“ (2014, Regie: Monika Treut), wo es um unkonventionelle Liebesbeziehungen geht, wobei es sich sozusagen um ein doppeltes Coming-out handelt: um ein Bekenntnis zu Pferde- und Mädchenfantasien. Zeh sieht im Pferdemädchen ebenfalls einen Ausdruck von Emanzipation von traditionellen Geschlechter-Stereotypen. Wenn junge Frauen den alten weißen Männern auf dem Hof an Kompetenz überlegen sind, so „erleben die Pferdefrauen den Geschlechterkampf Tag für Tag als gewonnenes Spiel“6.

Bei der Konjunktur des Pferdemädchens hat sicher auch die relativ junge Disziplin der Human-Animal-Studies ihren Anteil. Die Literaturwissenschaftlerin Elspeth Probyn plädiert beispielsweise dafür, das Pferd nicht „pop-psychoanalytisch“7 mit Phallus, Patriarchat und Herrschaft gleichzusetzen, sondern es als Teil und sozialen Akteur von nichthierarchischen Gesellschaftsstrukturen zu sehen. ${ }^{8}$ Sie interpretiert die Gemeinschaft der Pferdemädchen sogar als gelebte Utopie. Die deutsche Kulturanthropologin Verena Pohl ebenso wie die schwedische Ethnologin Karoliina Ojanen sehen Reitstallkulturen in vergleichbarer Weise als Räume von „alternative styles of femininity" " . Zweifellos steckt in dieser Heroisierung des Pferdemädchens auch eine Portion Idealismus. In jedem Fall aber ist die Figur inzwischen an Emanzipationsbewegungen anschließbar. 


\section{$\leftarrow$ Tweet}

\section{Judith Holofernes $\$$}

@jholofernes

\section{,Ruhig, Brauner! Wendy- Leser gegen Nazis' \#unteilbar}

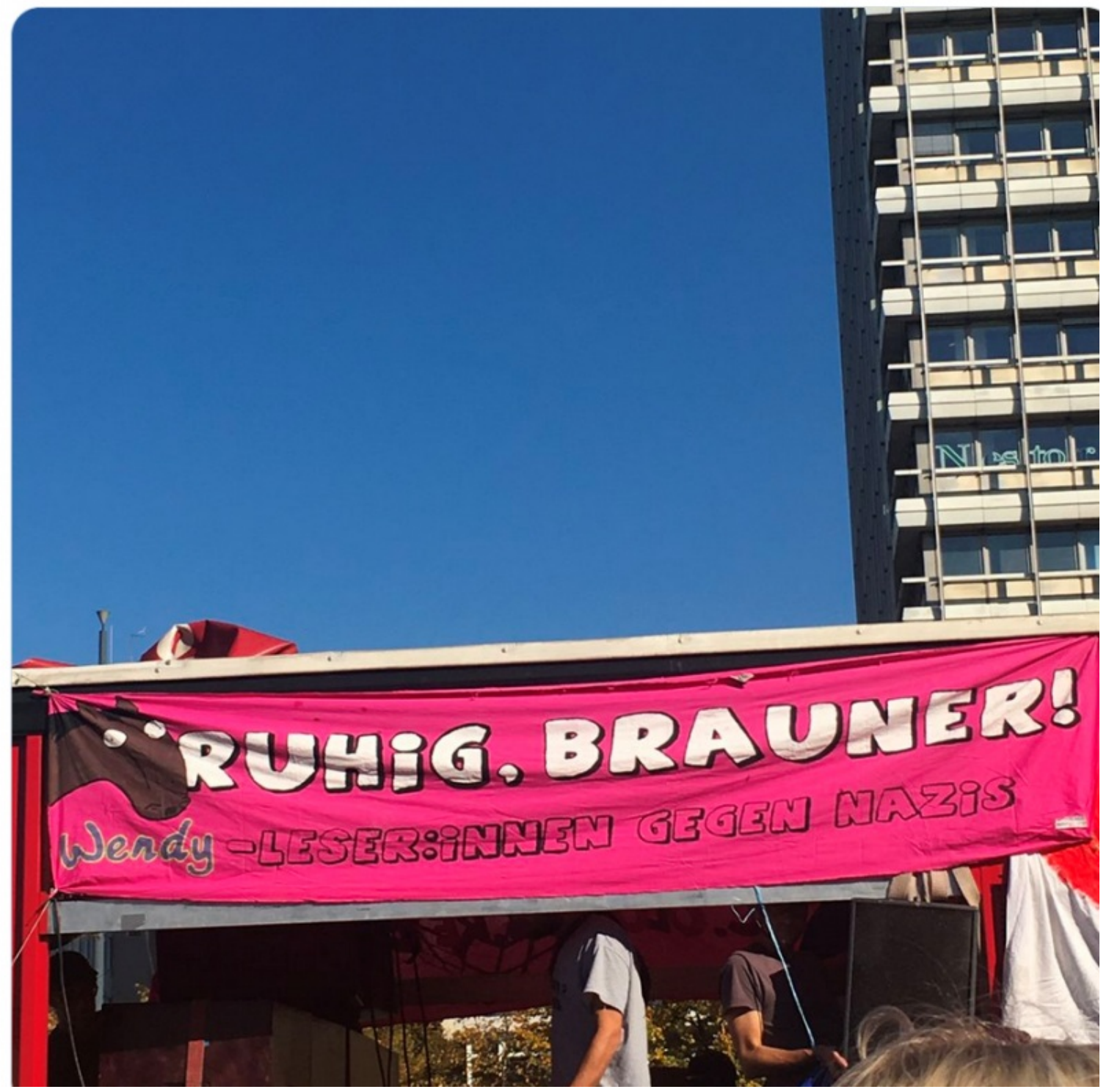

Pop-Referenz auf einer \#wirsindmehr-Demonstration 2018, ein Tweet der Sängerin Judith Holofernes (Screenshot aufgerufen am 18.09.2020)

\section{Pop-Feminismus}

Der jüngere Trend ist jedoch mit Emanzipation noch nicht hinreichend erklärt. Das zeigt ein Beispiel aus der jüngeren Geschichte, bei dem ein US-amerikanisches Pferdemädchen zu einer politischen Ikone wurde: Auf einer Demonstration gegen Polizeigewalt in Oakland anlässlich der Ermordung von George Floyd ritt Brianna Noble auf ihrem Pferd Dapper Dan durch die Straßen. Der Gefährte trug auf seinem Hinterteil ein Schild mit der Aufschrift „Black Lives Matter“. Bilder von den beiden 
gingen viral, worüber u. a. der britische Guardian berichtete. Sie ist nicht die einzige junge Frau, die aktuellen sozialen Protesten ein Gesicht gibt, allerdings bislang die einzige auf einem Pferd.

\section{Protesting from a pedestal: ‘No one can ignore a black woman on a horse'}

\section{Brianna Noble didn't plan to ride her horse into the George Floyd protest in Oakland, but it was her way of doing something positive}

l

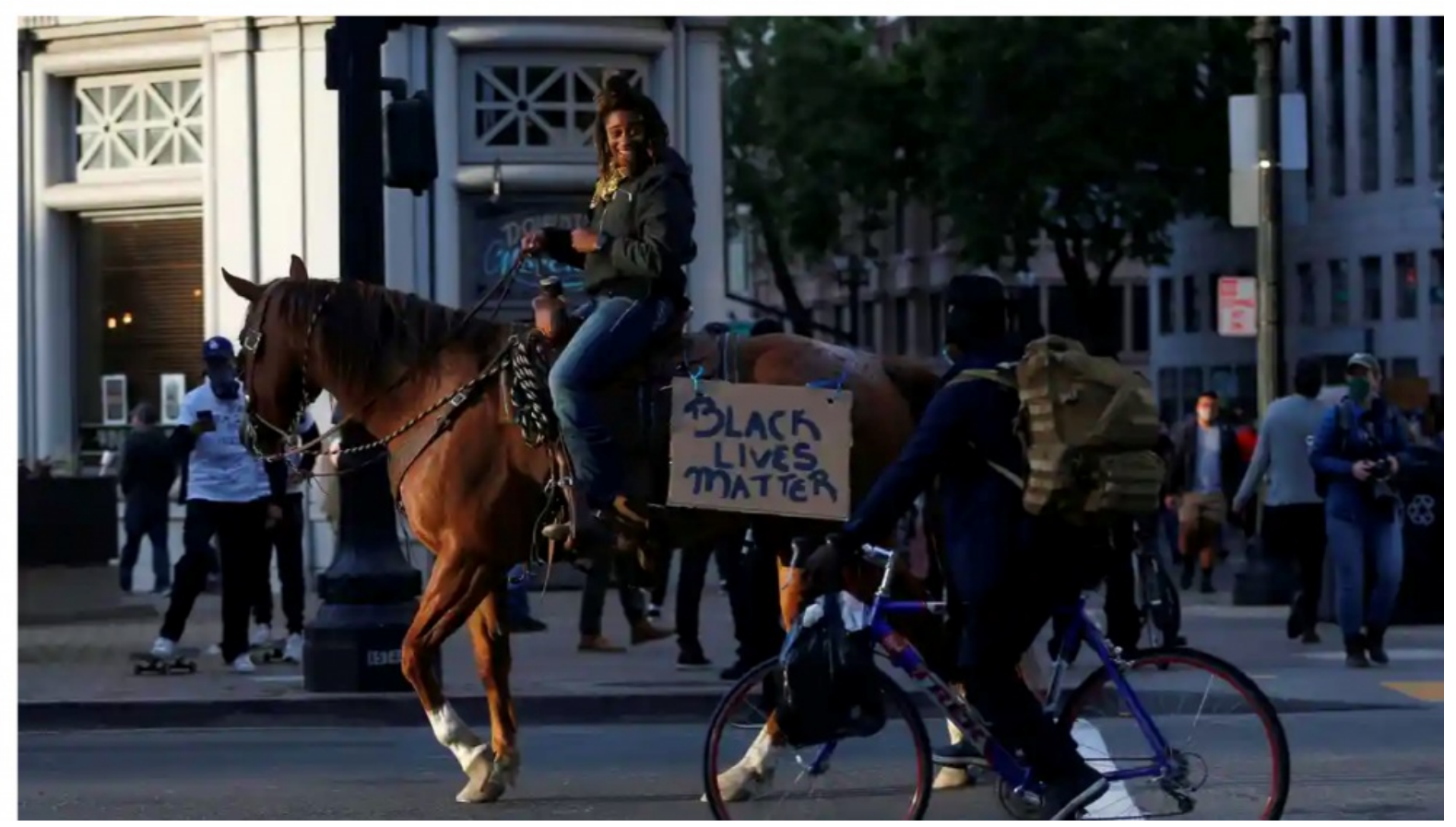

Guardian-Bericht über Brianna Noble auf Dapper Dan (Screenshot von The Guardian, aufgerufen am 17.09.2020)

Erst durch den Pop- und Netzfeminismus mit seiner typischen Ambivalenz von Affirmation und Subversion wurde es innerhalb der legitimen Geschmackskultur möglich, sich als Pferdemädchen zu outen. Pop-Feministinnen zeichnet genau jene Widersprüchlichkeit von Selbstverwirklichung und Selbstoptimierung, Body-Positive und Kosmetik, Schönheitswahn und Befreiung von demselben aus, die auch typisch für das Pferdemädchen ist. Anders ausgedrückt: Urbane junge Frauen dürfen sich heutzutage auf Pferden inszenieren, ohne zwangsläufig als apolitisch, heteronormativ und konservativ zu gelten. Die Mädchen, die in den sozialen Medien ein öffentliches Forum finden, sind Botschafterinnen eines veränderten Umweltbewusstseins, sie stehen für Anti-Rassismus, Einfühlsamkeit, Rücksichtnahme und Ressourcenschonung innerhalb eines Sozialwesens, in dem Natur nicht mehr länger in Opposition zu Gesellschaft steht.

\section{Inflation des Diminutiv}


Die Apologet*innen der Pferdemädchen-Kultur sind dabei nach wie vor in der Defensive. So ist bei Raulff der Diminutiv auffällig, er spricht von „kleinen Amazonen“ und „kleinen Mädchen“, womit er die Figur verniedlichend von anderen Zeitdiagnosen abgrenzt. Zu groß ist anscheinend die Gefahr, die hippologische Geschichtsschreibung durch das Mädchenhafte zu banalisieren. Auch Zeh distanziert sich letztlich vom Pferdemädchen, genauer genommen von der Populärkultur, was letztlich dasselbe ist. Sie sieht populärkulturelle Mythen als hinderlich für eine wie auch immer zu fassende authentische Mensch-Tier-Beziehung. Tatsächlich aber gibt es im Konsumkapitalismus, wo jedes Gefühl untrennbar mit medialen Bildern verbunden ist, keine Beziehung zum Pferd jenseits dieser Mythen. Hierfür ist das Phänomen des Pferdeflüsterns das beste Beispiel: „Horsemanship“, wie es auch genannt wird, ist als eine allgemein anerkannte Schule des Pferdetrainings durch den berühmten Film dennoch immer auch Pop. Mit der Tiertrainerin und Literaturwissenschaftlerin Vicki Hearne ist zu bemerken, dass es nicht darum geht, die populärkulturellen Narrative, mit denen man Problem-Pferde zu verstehen versucht, abzuschaffen, sondern das für das individuelle Pferd passende Narrativ zu finden. ${ }^{10}$

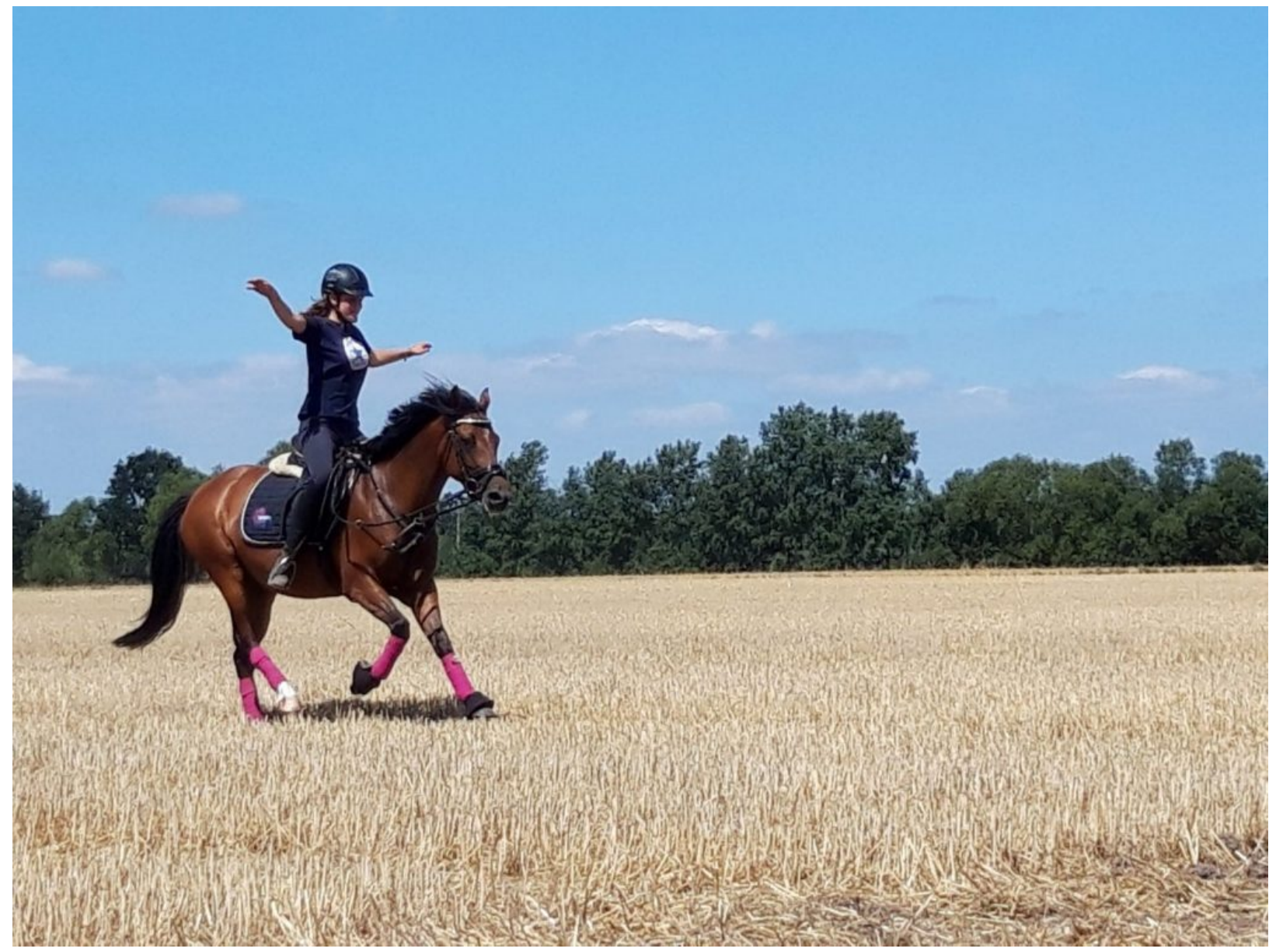

Romantik und Pop: Galopp übers Stoppelfeld, (C) Nutzungsrecht Anja Schwanhäußer

\section{Schluss}


Pferdemädchen werden also trotz ihrer Konjunktur nach wie vor belächelt. Dies hat vor allem damit zu tun, dass die Populär- und Konsumkultur nach wie vor nicht als kreativ und bedeutsam anerkannt wird, wie die Modetheoretikerin Diana Weis in ihrem jüngst erschienenen Buch „Modebilder“ kritisiert. ${ }^{11}$ Wenn infolgedessen die Populärkultur abgewertet wird, wird zugleich auch die Mädchenwelt abgewertet, und umgekehrt. Es ist aber an der Zeit, Pferdemädchen als eigene Jugendsubkultur wahrzunehmen. Nicht nur, um Mädchen, sondern auch, um die mädchenhafte Gefühlsstruktur unserer Zeit besser zu verstehen.

\section{References}

1. Raulff, Ulrich (2015): Das letzte Jahrhundert der Pferde. Geschichte einer Trennung. München: Beck. https://doi.org/10.17104/9783406682452

2. Zeh, Juli (2019): Gebrauchsanweisung für Pferde. München: Piper, S. 9.

3. Freud, Anna (1989): Normality and Pathology in Childhood: Assessments of Development. New York: Routledge, S. 20.

4. Vgl. Illouz, Eva (2018): Wa(h)re Gefühle - Authentizität im Konsumkapitalismus. Frankfurt/Main: Suhrkamp.

5. Raulff, S. 296.

6. Zeh, S. 100.

7. Probyn, Elspeth (1993): Girls and Girls and Girls and Horses: Queer Images of Singularity and Desire. In: Tessera. Vol. 15 (Winter), S. 22-29, hier: S. 25. Siehe auch: Halley, Jean O’Malley (2019): Horse Crazy: Girls and the Lives of Horses. Athens, Georgia: Georgia Press. https://doi.org/10.25071/1923-9408.24995

8. Probyn, S. $24 \mathrm{f}$.

9. Ojanen, Karoliina (2012): ,You Became Someone'. Social Hierarchies in Girls' Communities at Riding Stables. In: Young 20(2), S. 137-156, hier: S. 145. Pohl, Verena (2010): Pferdeliebe auf dem x-Chromosom. In: Regina Bendix, Michaela Fenske (Hg.): kulturen. Sonderheft: Pferd - Kultur - Niedersachsen, S. 67-79. https://doi.org/10.1177/110330881202000202

10. Hearne, Vicki (2007): Adam's task. Calling Animals by Name. New York: Skyhorse, S. 126.

11. Weis, Diana (2020): Modebilder. Berlin: Wagenbach, S. 33.

SUGGESTED CITATION: Schwanhäußer, Anja: Horse Crazy - Gefühle im Konsumkapitalismus, in: KWI-BLOG, [https://blog.kulturwissenschaften.de/pferdemaedchen/l, 23.09.2020

DOI: https://doi.org/10.37189/kwi-blog/20200923-0900 


\section{DuEPublico}

Duisburg-Essen Publications online

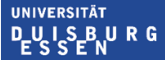

offen im Denken

\section{Ub $\begin{aligned} & \text { universitäts } \\ & \text { bibliothek }\end{aligned}$}

Dieser Text wird über DuEPublico, dem Dokumenten- und Publikationsserver der Universität Duisburg-Essen, zur Verfügung gestellt. Die hier veröffentlichte Version der EPublikation kann von einer eventuell ebenfalls veröffentlichten Verlagsversion abweichen.

DOI: $\quad$ 10.37189/kwi-blog/20200923-0900

URN: urn:nbn:de:hbz:464-20200923-091457-5

Alle Rechte vorbehalten. 